\title{
FUTURE PLANS FOR THE SMALL ISOCHRONOUS RING*
}

\author{
E. Pozdeyev, JLab, Newport News, VA 23606, USA \\ F. Marti, R. C. York, MSU, East Lansing, MI 48824-1321, USA \\ J. Rodriguez, CERN, Geneva, Switzerland
}

\begin{abstract}
The Small Isochronous Ring (SIR) has been operational at Michigan State University since December 2003. It has been used for experimental studies of the beam dynamics in high-intensity isochronous cyclotrons and synchrotrons at the transition energy. Operational experience with SIR has demonstrated that the ring can be successfully used to study space charge effects in accelerators. The low velocity of beam particles in the ring allowed longitudinal profile measurements with an accuracy that would be difficult to achieve in full-size accelerators. The experimental data obtained in the ring was used for validation of the multiparticle, space-charge codes CYCO and WARP3D. Encouraged by the success of SIR in the isochronous regime, we consider options for expanding the scope of the beam physics studied in the ring. In this paper, we outline possible future experiments and discuss required modifications of the ring optics and hardware.
\end{abstract}

\section{INTRODUCTION}

Operational experience with SIR [1] has demonstrated that low-energy, light ions can be successfully used for experimental studies of space charge effects and for benchmarking multi-particle codes. Several experiments, including the characterization of the longitudinal beam breakup in the isochronous regime and the benchmarking of computer codes CYCO and WARP3D, were successfully conducted using SIR.

SIR consists of four flat-field dipoles with edge focusing providing both vertical focusing and the isochronism in the ring. The ring includes two types of quadrupole correctors that allow variation of the betatron tunes and the compaction factor by approximately \pm 0.1 . The variance of the two types of quadrupoles does not, however, allow for the independent adjustment of each tune and the compaction factor. Because the ring is isochronous, it does not require nor include an RF system. The simplicity of the lattice design allowed us to build the ring in a short time with limited resources without compromising the goal of experimental studies of space charge effects in the isochronous regime.

Verified by the experimental data obtained in SIR [1], longitudinal and radial space charge forces cause beam bunches to break into small clusters in a few turns at beam intensities corresponding to a Laslett tune shift of only a few per cent. The longitudinal breakup at low intensities

\footnotetext{
* Work supported by NSF Grant \#PHY-0110253 and DOE Contract
} DE-AC05-84ER40150 significantly complicates studies of transverse space charge effects.

\section{UPGRADE OBJECTIVES}

The SIR upgrade is designed to explore transverse and longitudinal space charge effects in a high-intensity regime corresponding to a Laslett tune shift of $50 \%$. Space charge phenomena of primary interest for the upgrade include:

- Fast and slow traversal of half-integer and higher order resonances by the intense beam

- Beam loss and beam life-time at high beam intensities

- Tune dependence of the beam parameters and beam life-time

- Dynamics of bunch head and tail

- Beam envelope instability.

\section{UPGRADE LAYOUT AND DESIGN FEATURES}

\section{Upgrade Layout and Main Parameters}

To be able to address the extended scope of research, the upgraded ring will operate in a non-isochronous regime. A significant variation of the compaction factor must be accompanied by a shift of the horizontal betatron tune, which, in turn, requires modification of the ring optics. At least two additional sets of quadrupoles are required to increase the horizontal focusing and to control the location of the vertical tune on the tune diagram. The existing gradient correctors situated in the dipole magnets can be used to adjust the compaction factor of the ring independently from the betatron tunes. Two sets of sextupoles can be also included to compensate the natural chromaticity of the betatron tunes.

The SIR upgrade will utilize the existing dipole magnets and vacuum chambers of the straight sections. To accommodate the additional quadrupoles, the length of the straight sections between the dipoles will be increased by $50 \mathrm{~cm}$. Two $10 \mathrm{~cm}$-long quadrupoles of opposite polarity will be inserted in each drift as shown in Figure 1. The required field gradient produced by the quadrupoles is of the order of 40 Gauss $/ \mathrm{cm}$. Table 1 shows the upgrade parameters and those of the existing ring. The optical functions of the upgrade are shown in Figure 2. 


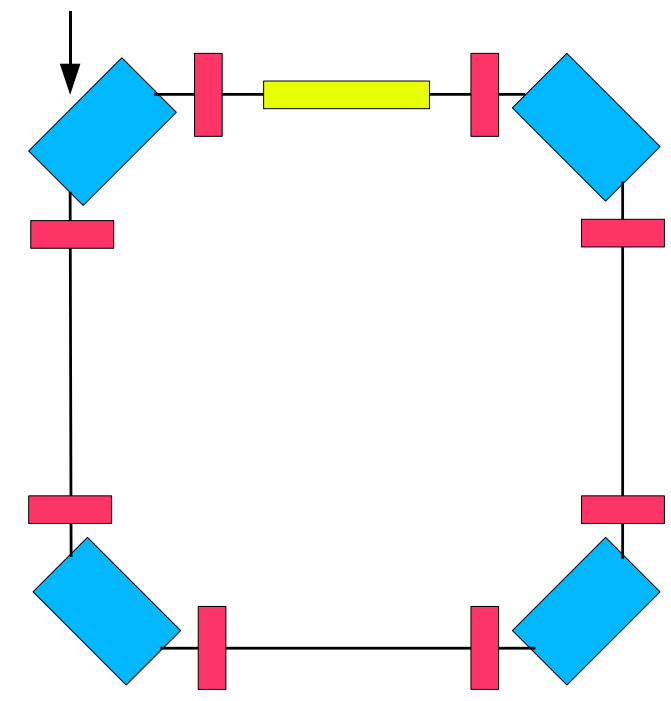

Figure 1: SIR upgrade utilizes existing dipole magnets. The length of the drifts is increased by $50 \mathrm{~cm}$ to accommodate 8 new quadrupoles, shown by red rectangles. The yellow rectangle schematically shows the RF drift tube.

Table 1: Parameters of the existing ring and proposed upgrade

\begin{tabular}{|l|c|c|}
\hline Parameters & SIR & Upgrade \\
\hline Max. energy $(\mathrm{keV})$ & 30 & 30 \\
$\nu_{x}, \nu_{y}$ & $1-1.2$ & $1.35-1.85$ \\
$\alpha_{p}$ & $0.9-1.1$ & $0.45-0.95$ \\
$\nu_{s}\left(\right.$ for $\left.\alpha_{p}=0.7, V_{\mathrm{rf}}=100 \mathrm{~V}\right)$ & 0.0 & $8.7 \mathrm{e}-3$ \\
Beam peak current $(\mu \mathrm{A})$ & $0-80$ & $0-230$ \\
Laslett tune shift, $\delta \nu / \nu_{0}$ & $0-20 \%$ & $0-50 \%$ \\
Circumference $(\mathrm{m})$ & 6.6 & 8.6 \\
\hline
\end{tabular}

\section{RF System}

The upgrade will include an RF system to provide longitudinal focusing and to maintain the longitudinal beam size. In its simplest form, the RF system would consist of a single drift tube driven by a sine wave generator. The maximum energy gain produced by the accelerating tube is given by

$$
q V_{\mathrm{eff}}=2 q V_{0} \sin \left(\frac{\pi h l}{C}\right)
$$

where $V_{0}$ is the maximum potential on the tube, $h$ is the harmonic number, $l$ is the cylinder length, and $C$ is the ring circumference. If $V_{0}$ is $100 \mathrm{~V}, h$ is $1, l$ is $60 \mathrm{~cm}$, and $C$ is $8.6 \mathrm{~m}$, the effective voltage produced by the tube is equal to 44 Volts. A more complicated system including higher harmonics of the revolution frequency to compensate the longitudinal space charge force at the bunch ends will be considered.

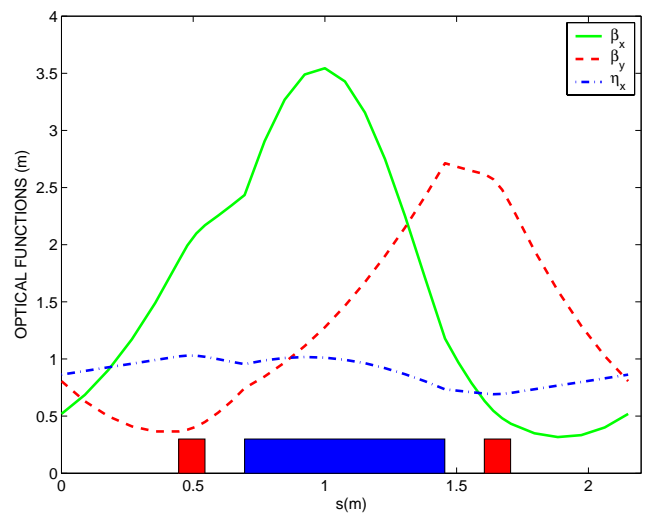

Figure 2: The optical functions of the SIR upgrade shown for a single period of the ring.

\section{Diagnostics}

A high-resolution energy analyzer similar to that developed at the University of Maryland [3] will be a valuable addition to the existing phosphor screen and Faraday Cup currently used to measure the transverse and longitudinal profiles of the extracted beam.

\section{LASLETT TUNE-SHIFT AND STABILITY OF BEAM ENVELOPE IN SIR UPGRADE} [2]:

The equations of the beam envelopes $X(s)$ and $Y(s)$ are

$$
\begin{aligned}
& X^{\prime \prime}+k_{x 0} X-\frac{2 K}{X+Y}-\frac{\epsilon_{x}^{2}}{X^{3}}=0 \\
& Y^{\prime \prime}+k_{y 0} Y-\frac{2 K}{X+Y}-\frac{\epsilon_{x}^{2}}{Y^{3}}=0,
\end{aligned}
$$

where $k_{x 0}$ and $k_{y 0}$ are the external focusing functions and $K$ is the generalized perveance. Equations (2)-(3) were integrated numerically to obtain the beam envelopes and the betatron tunes. Figure 3 shows the beam tunes on the tune diagram for different beam currents.

The linearized equations for small envelope perturbations are [2]

$$
\begin{aligned}
\xi^{\prime \prime}+a_{1}(s) \xi+a_{0}(s) \eta & =0 \\
\eta^{\prime \prime}+a_{2}(s) \eta+a_{0}(s) \xi & =0
\end{aligned}
$$

where $\xi$ and $\eta$ are small deviations from the matched beam envelopes $X$ and $Y . a_{0}, a_{1}$ and $a_{2}$ are periodic functions depending on the external focusing, beam current, and the matched beam envelopes. Numerical integration of (4)-(5) for four characteristic functions with the initial conditions $\left(\xi, \xi^{\prime}, \eta, \eta^{\prime}\right)_{1,2,3,4}=$ $(1,0,0,0),(0,1,0,0),(0,0,1,0),(0,0,0,1)$ yields the matrix of the transformation given by (4)-(5). Oscillations of mismatched envelopes are stable if the absolute value of the matrix eigen values is equal to 1 . Table 2 shows the absolute value of the four eigen values vs. peak beam 


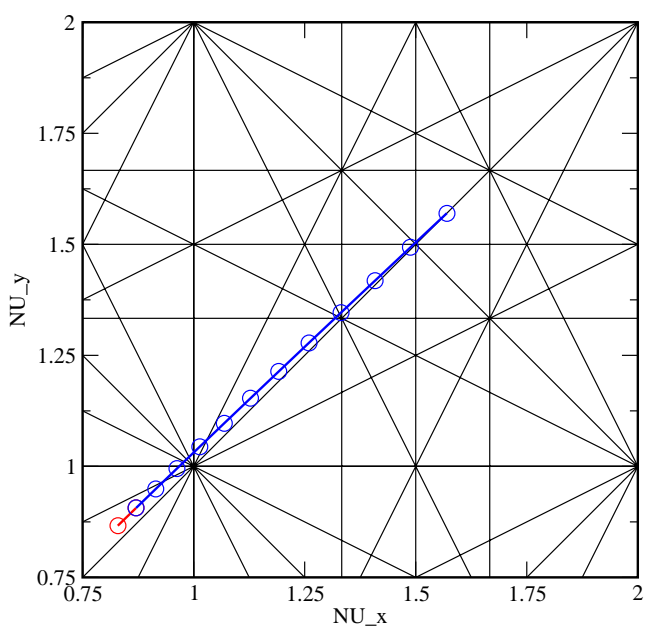

Figure 3: Beam tunes calculated from the numerical solution of the system (2)-(3) for different beam currents. The bare tunes are $\nu_{x}=\nu_{y}=1.57$. Each circle corresponds to a beam current increment of $20 \mu \mathrm{A}$. The last, red point corresponds to the beam current at which the beam envelope instability occurs.

current for the $20 \mathrm{keV}, 50 \pi \cdot \mathrm{mm} \cdot \mathrm{mrad}, \mathrm{H}_{2}^{+}$beam. The oscillations are stable if the peak beam current does not exceed $220 \mu \mathrm{A}$. This result was confirmed by a direct tracking of mismatched beam envelopes. Figure 4 shows the behavior of mismatched beam envelopes for a beam current of $220 \mu \mathrm{A}$ and $240 \mu \mathrm{A}$.

Table 2: The absolute value of the four eigen values of the mismatched beam envelope oscillations vs. peak beam current. The oscillations are stable if the beam current is below $230 \mu \mathrm{A}$

\begin{tabular}{|l|c|c|c|c|}
\hline $\mathbf{I}_{\text {peak }}(\mu \mathbf{A})$ & $\left|\lambda_{1}\right|$ & $\left|\lambda_{2}\right|$ & $\left|\lambda_{3}\right|$ & $\left|\lambda_{4}\right|$ \\
\hline $0-220$ & 1 & 1 & 1 & 1 \\
230 & 1 & 1 & 1.06 & 0.94 \\
240 & 1 & 1 & 1.14 & 0.88 \\
250 & 1 & 1 & 1.16 & 0.86 \\
\hline
\end{tabular}

\section{CONCLUSSIONS AND FUTURE STUDIES}

This paper outlines the objectives of the SIR upgrade and presents a candidate for the upgrade optics. Additional work is required to address the following questions in detail:

- Analysis of the longitudinal beam dynamics with the space charge and longitudinal focusing

- Energy-phase and dispersion matching to the ring

- Efficacy of sextupole correctors in the ring
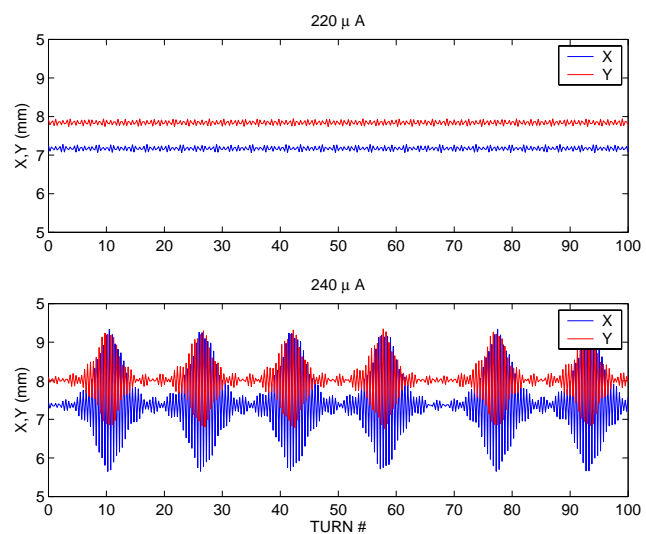

Figure 4: Beam envelopes obtained by numerical integration of the system (2)-(3) for a beam current of $220 \mu \mathrm{A}$ and $240 \mu \mathrm{A}$ vs. turn number. The envelopes are plotted once per each period of the ring. The initial size of the envelopes $X$ and $Y$ was larger than the matched beam size by $1 \%$.

\section{ACKNOWLEDGEMENT}

The authors are thankful to Alexei Fedotov for his interest in the work and useful discussions.

\section{REFERENCES}

[1] E. Pozdeyev, F. Marti, J. Rodriguez, and R.C. York, ”Experimental Results from the Small Isochronous Ring at Michigan State University", these proceedings.

[2] M. Reiser, "Theory and design of charged particle beams", John Wiley and Sons, Inc., New York (1994).

[3] Y. Zou, et. al., "Compact high-resolution retarding field energy analyzer for space-charge-dominated electron beams", PRST-AB, V. 5, 072801 (2002) 